\title{
ON CERTAIN PROPERTIES OF BRANCHING COEFFICIENTS FOR AFFINE LIE ALGEBRAS
}

\author{
M. ILYIN, P. KULISH, AND V. LYAKHOVSKY
}

\begin{abstract}
It is demonstrated that the decompositions of integrable highest weight modules of a simple Lie algebra (classical or affine) with respect to its reductive subalgebra obey a set of algebraic relations leading to recursive properties for the corresponding branching coefficients. These properties are encoded in a special element $\Gamma_{\mathfrak{g} \supset \mathfrak{a}}$ of the formal algebra $\mathcal{E}_{\mathfrak{a}}$ that describes the injections $\mathfrak{a} \rightarrow \mathfrak{g}$ and is called a fan. In the simplest case where $\mathfrak{a}=\mathfrak{h}(\mathfrak{g})$, the recursion procedure generates the weight diagram of a module $L_{\mathfrak{g}}$. When the recursion described by a fan is applied to highest weight modules, it provides a highly efficient tool for explicit calculations of branching coefficients.
\end{abstract}

\section{$\S 1$. INTRODUCTION}

We consider integrable modules $L^{\mu}$ of an affine Lie algebra $\mathfrak{g}$ with the highest weight $\mu$ and reduced modules $L_{\mathfrak{g} \downarrow \mathfrak{a}}^{\mu}$ with respect to a reductive subalgebra $\mathfrak{a} \subset \mathfrak{g}$. In particular, when the Cartan subalgebra $\mathfrak{a}=\mathfrak{h}$ is studied, the branching coefficients indicate the dimensions of the weight subspaces and thus describe the module. String functions and branching coefficients of the affine Lie algebra pairs (for example, $A_{n}^{(1)} \subset A_{n-p-1}^{(1)} \oplus A_{p}^{(1)}$ ) arise in the computation of the local state probabilities for solvable models on square lattices [1. Irreducible highest weight modules with dominant integral weights arise also in applications of the quantum inverse scattering method [2] where solvable spin chains are studied in the framework of the AdS/CFT correspondence conjecture of superstring theory (see [3, 4]).

There are different ways to find branching coefficients. One can use the BernsteinGel'fand-Gel'fand resolution [7] (for Kac-Moody algebras the algorithm was described in [5, 6]), the Schur function series [8, the BRST cohomology [9], Kac-Peterson formulas [5], or the combinatorial methods applied in $[10$. In this paper we want to obtain recursive formulas for weight multiplicities and branching coefficients by using a purely algebraic approach. From the Weyl-Kac character formula [5],

$$
\operatorname{ch} L^{\mu}(\mathfrak{g})=\frac{\sum_{w \in W} \epsilon(w) e^{w \circ(\mu+\rho)-\rho}}{\prod_{\alpha \in \Delta^{+}}\left(1-e^{-\alpha}\right)^{\operatorname{mult}(\alpha)}}
$$

we derive a special set of relations for branching coefficients. These relations can be used both to construct a representation and to reduce it with respect to a subalgebra $\mathfrak{a} \subset \mathfrak{g}$, i.e., to find the corresponding branching rules. Each relation of this set involves a finite collection of weights. Among them, it is always possible to fix the lowest one

2000 Mathematics Subject Classification. 17B10, 17B20.

Key words and phrases. Integrable highest weight modules, simple Lie algebra, reductive subalgebra, branching coefficients, fan, weight diagram.

The second author was supported by RFFI grant 09-01-00504.

The third author was supported by RFFI grant 09-01-00504 and the National Project RNP.2.1.1./1575. 
(with respect to the natural ordering of weights induced by the basic roots). Thus, it is possible to use the relations of the set as recursion relations for branching coefficients. It is demonstrated that branching is governed by a certain system of weights (called "the injection fan") that depends only on the algebra and the injection morphism and can be used to decompose highest weight modules.

For finite-dimensional classical Lie algebras, the case of regular injections was considered in [11, where recursion relations were constructed by using the properties of the Kostant-Heckman partition function. The same method was used for regular injections of affine Lie algebras [12. In the present study, we employ a different approach and find that for any reductive subalgebra $\mathfrak{a}$ of an affine Lie algebra $\mathfrak{g}$ such that $\mathfrak{h}_{\mathfrak{a}}^{*} \subset \mathfrak{h}_{\mathfrak{g}}^{*}$ and $\mathfrak{h}_{\mathfrak{a}}^{*} \subset \mathfrak{h}_{\mathfrak{g}}^{*}$, the branching coefficients obey a set of properties that give rise to recursion relations. These relations provide a compact and efficient method to construct the corresponding branching rules. The results are illustrated by examples.

\section{$\S 2$. BASIC DEFINITIONS AND RELATIONS}

Consider two affine Lie algebras $\mathfrak{g}$ and $\mathfrak{a}$ with the underlying finite-dimensional subalgebras $\mathfrak{g}$ and $\mathfrak{a}$ and an injection $\mathfrak{a} \longrightarrow \mathfrak{g}$ such that $\mathfrak{a}$ is a reductive subalgebra $\mathfrak{a} \subset \mathfrak{g}$ with correlated root spaces: $\mathfrak{h}_{\mathfrak{a}}^{*} \subset \mathfrak{h}_{\mathfrak{g}}^{*}$ and $\mathfrak{h}_{\mathfrak{a}}^{*} \subset \mathfrak{h}_{\mathfrak{g}}^{*}$.

The following notation will also be used:

$L^{\mu}\left(L_{\mathfrak{a}}^{\nu}\right)$ - the integrable module of $\mathfrak{g}$ with the highest weight $\mu$ (respectively, integrable $\mathfrak{a}$-module with the highest weight $\nu$ );

$r\left(r_{\mathfrak{a}}\right)$ - the rank of the algebra $\mathfrak{g}$ (respectively, $\mathfrak{a}$ );

$\Delta\left(\Delta_{\mathfrak{a}}\right)$ — the root system; $\Delta^{+}\left(\Delta_{\mathfrak{a}}^{+}\right)$- the positive root system (of $\mathfrak{g}$ and $\mathfrak{a}$, respectively);

$\operatorname{mult}(\alpha)\left(\operatorname{mult}_{\mathfrak{a}}(\alpha)\right)$ - the multiplicity of the root $\alpha$ in $\Delta$ (respectively, in $\left.\left(\Delta_{\mathfrak{a}}\right)\right)$;

$\stackrel{\Delta}{\Delta}\left(\AA_{\mathfrak{a}}\right)$ - the finite root system of the subalgebra $\mathfrak{g}$ (respectively, $\mathfrak{a}$ );

$\mathcal{N}^{\mu}\left(\mathcal{N}_{\mathfrak{a}}^{\nu}\right)$ - the weight diagram of the module $L^{\mu}$ (respectively, $L_{\mathfrak{a}}^{\nu}$ );

$W\left(W_{\mathfrak{a}}\right)$ - the corresponding Weyl group;

$C\left(C_{\mathfrak{a}}\right)$ - the fundamental Weyl chamber;

$\rho\left(\rho_{\mathfrak{a}}\right)$ - the Weyl vector;

$\epsilon(w):=\operatorname{det}(w)$

$\alpha_{i}\left(\alpha_{(\mathfrak{a}) j}\right)$ - the $i$ th (respectively, $j$ th) basic root for $\mathfrak{g}($ respectively, $\mathfrak{a}) ; i=0, \ldots, r$

$\left(j=0, \ldots, r_{\mathfrak{a}}\right)$;

$\delta$ - the imaginary root of $\mathfrak{g}$ (and of $\mathfrak{a}$ if any);

$\alpha_{i}^{\vee}\left(\alpha_{(\mathfrak{a}) j}^{\vee}\right)$ - the basic coroot for $\mathfrak{g}$ (respectively, $\left.\mathfrak{a}\right), i=0, \ldots, r\left(j=0, \ldots, r_{\mathfrak{a}}\right)$;

$\stackrel{\circ}{\xi}, \stackrel{\circ}{\xi}_{(\mathfrak{a})}$ - the finite (classical) part of the weight $\xi \in P$ (respectively, $\xi_{(\mathfrak{a})} \in P_{\mathfrak{a}}$ );

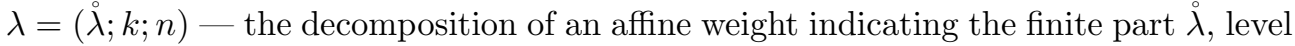

$k$, and grade $n$.

$P$ (respectively, $\left.P_{\mathfrak{a}}\right)$ - the weight lattice;

$M$ (respectively, $M_{\mathfrak{a}}$ )

$$
:=\left\{\begin{array}{c}
\sum_{i=1}^{r} \mathbf{Z} \alpha_{i}^{\vee}\left(\text { respectively, } \sum_{i=1}^{r} \mathbf{Z} \alpha_{(\mathfrak{a}) i}^{\vee}\right) \text { for untwisted algebras or } A_{2 r}^{(2)}, \\
\sum_{i=1}^{r} \mathbf{Z} \alpha_{i}\left(\text { respectively, } \sum_{i=1}^{r} \mathbf{Z} \alpha_{(\mathfrak{a}) i}\right) \text { for } A_{r}^{(u \geq 2)} \text { and } A \neq A_{2 r}^{(2)}
\end{array}\right\} ;
$$

$\mathcal{E}\left(\mathcal{E}_{\mathfrak{a}}\right)$ - the group algebra of the group $P$ (respectively, $P_{\mathfrak{a}}$ );

$\Theta_{\lambda}:=e^{-\frac{|\lambda|^{2}}{2 k} \delta} \sum_{\alpha \in M} e^{t_{\alpha} \circ \lambda}$ - the classical theta-function; 
$\Theta_{(\mathfrak{a}) \nu}:=e^{-\frac{|\nu|^{2}}{2 k_{\mathfrak{a}}} \delta} \sum_{\beta \in M_{\mathfrak{a}}} e^{t_{\beta} \circ \nu}$; notice that, when an injection is considered, the level $k_{\mathfrak{a}}$ must be correlated with the corresponding rescaling of roots;

$$
\begin{aligned}
A_{\lambda} & :=\sum_{s \in \dot{W}} \epsilon(s) \Theta_{s \circ \lambda} \quad\left(\text { respectively, } A_{(\mathfrak{a}) \nu}:=\sum_{s \in \mathscr{W}_{\mathfrak{a}}} \epsilon(s) \Theta_{(\mathfrak{a}) s \circ \nu}\right) \\
\Psi^{(\mu)} & :=e^{\frac{|\mu+\rho|^{2}}{2 k} \delta-\rho} A_{\mu+\rho}=e^{\frac{|\mu+\rho|^{2}}{2 k} \delta-\rho} \sum_{s \in \dot{W}} \epsilon(s) \Theta_{s \circ(\mu+\rho)} \\
& =\sum_{w \in W} \epsilon(w) e^{w \circ(\mu+\rho)-\rho}-\text { the singular weight element for the } \mathfrak{g} \text {-module } L^{\mu}
\end{aligned}
$$

$$
\begin{aligned}
\Psi_{(\mathfrak{a})}^{(\nu)} & :=e^{\frac{\left|\nu+\rho_{\mathfrak{a}}\right|^{2}}{2 k_{\mathfrak{a}}} \delta-\rho_{\mathfrak{a}}} A_{(\mathfrak{a}) \nu+\rho_{\mathfrak{a}}}=e^{\frac{\left|\nu+\rho_{\mathfrak{a}}\right|^{2}}{2 k_{\mathfrak{a}}} \delta-\rho_{\mathfrak{a}}} \sum_{s \in W_{\mathfrak{a}}} \epsilon(s) \Theta_{(\mathfrak{a}) s \circ\left(\nu+\rho_{\mathfrak{a}}\right)} \\
& =\sum_{w \in W_{\mathfrak{a}}} \epsilon(w) e^{w \circ\left(\nu+\rho_{\mathfrak{a}}\right)-\rho_{\mathfrak{a}}}-\text { the corresponding singular weight element } \\
& \text { for the } \mathfrak{a} \text {-module } L_{\mathfrak{a}}^{\nu} ;
\end{aligned}
$$

$\widehat{\Psi^{(\mu)}}\left(\widehat{\Psi_{(\mathfrak{a})}^{(\nu)}}\right)$ - the set of singular weights $\xi$ in $P$ (respectively, in $P_{\mathfrak{a}}$ ) for the module $L^{\mu}$ (respectively, $L_{\mathfrak{a}}^{\nu}$ ) with the coordinates

$$
\left.(\stackrel{\circ}{\xi}, k, n, \epsilon(w(\xi)))\right|_{\xi=w(\xi) \circ(\mu+\rho)-\rho}
$$

(respectively, $\left.\left(\dot{\xi}, k, n, \epsilon\left(w_{a}(\xi)\right)\right)\right|_{\xi=w_{a}(\xi) \circ\left(\nu+\rho_{a}\right)-\rho_{a}}$ ) (this set is similar to $P_{\text {nice }}^{\prime}(\mu)$ in [6]); $m_{\xi}^{(\mu)}\left(m_{\xi}^{(\nu)}\right)$ - the multiplicity of the weight $\xi$ in $P$ (respectively, in $P_{\mathfrak{a}}$ ) in the module $L^{\mu}\left(\right.$ respectively, $\left.\xi \in L_{\mathfrak{a}}^{\nu}\right)$;

$\operatorname{ch}\left(L^{\mu}\right)\left(\right.$ respectively, $\left.\operatorname{ch}\left(L_{\mathfrak{a}}^{\nu}\right)\right)$ - the formal character of $L^{\mu}$ (respectively, $L_{\mathfrak{a}}^{\nu}$ );

$$
\operatorname{ch}\left(L^{\mu}\right)=\frac{\sum_{w \in W} \epsilon(w) e^{w \circ(\mu+\rho)-\rho}}{\prod_{\alpha \in \Delta^{+}}\left(1-e^{-\alpha}\right)^{\text {mult }(\alpha)}}=\frac{\Psi^{(\mu)}}{\Psi^{(0)}}-\text { the Weyl-Kac formula. }
$$$$
R:=\prod_{\alpha \in \Delta^{+}}\left(1-e^{-\alpha}\right)^{\operatorname{mult}(\alpha)}=\Psi^{(0)}
$$

(respectively, $\left.R_{\mathfrak{a}}:=\prod_{\alpha \in \Delta_{\mathfrak{a}}^{+}}\left(1-e^{-\alpha}\right)^{\text {mult }_{\mathfrak{a}}(\alpha)}=\Psi_{\mathfrak{a}}^{(0)}\right)$ - the denominator.

\section{§3. Anomalous multiplicities and ReCursion Relations}

For the injection $\mathfrak{a} \longrightarrow \mathfrak{g}$, consider the reduced module

$$
L_{\mathfrak{g} \downarrow \mathfrak{a}}^{\mu}=\bigoplus_{\nu \in P_{\mathfrak{a}}^{+}} b_{\nu}^{(\mu)} L_{\mathfrak{a}}^{\nu}
$$

with the branching coefficients $b_{\nu}^{(\mu)}$. The character reduction

$$
\pi_{\mathfrak{a}} \circ\left(\operatorname{ch} L_{\mathfrak{g}}^{\mu}\right)=\sum_{\nu \in P_{\mathfrak{a}}^{+}} b_{\nu}^{(\mu)} \operatorname{ch} L_{\mathfrak{a}}^{\nu}
$$

involves the projection operator $\pi_{\mathfrak{a}}: P \longrightarrow P_{\mathfrak{a}}$.

We consider the numerator on the right-hand side of (3) and introduce the numbers $k_{\lambda}^{(\mu)}$, called the anomalous branching coefficients, which are the multiplicities of the submodules $L_{\mathfrak{a}}^{\nu}$ times the determinants $\epsilon(w)$ contained in $\Psi_{(\mathfrak{a})}^{(\nu)}$ :

$$
\sum_{\nu \in P_{\mathfrak{a}}} b_{\nu}^{(\mu)} \Psi_{(\mathfrak{a})}^{(\nu)}=\sum_{\lambda \in P_{\mathfrak{a}}} k_{\lambda}^{(\mu)} e^{\lambda}
$$


The denominator identity can be applied to redress relation (3),

$$
\frac{\pi_{\mathfrak{a}} \circ\left(\sum_{w \in W} \epsilon(w) e^{w \circ(\mu+\rho)-\rho}\right)}{\pi_{\mathfrak{a}} \circ\left(\prod_{\alpha \in \Delta^{+}}\left(1-e^{-\alpha}\right)^{\operatorname{mult}(\alpha)}\right)}=\sum_{\nu \in P_{\mathfrak{a}}^{+}} b_{\nu}^{(\mu)} \frac{\sum_{w \in W_{\mathfrak{a}}} \epsilon(w) e^{w \circ\left(\nu+\rho_{\mathfrak{a}}\right)-\rho_{\mathfrak{a}}}}{\prod_{\beta \in \Delta_{\mathfrak{a}}^{+}}\left(1-e^{-\beta}\right)^{\text {mult }_{\mathfrak{a}}(\beta)}},
$$

and to rewrite it in the form

$$
\begin{aligned}
\pi_{\mathfrak{a}} \circ & \left(\sum_{w \in W} \epsilon(w) e^{w \circ(\mu+\rho)-\rho}\right) \\
& =\frac{\pi_{\mathfrak{a}} \circ\left(\prod_{\alpha \in \Delta^{+}}\left(1-e^{-\alpha}\right)^{\operatorname{mult}(\alpha)}\right)}{\prod_{\beta \in \Delta_{\mathfrak{a}}^{+}}\left(1-e^{-\beta}\right)^{\operatorname{mult}_{\mathfrak{a}}(\beta)}} \sum_{\nu \in P_{\mathfrak{a}}^{+}} b_{\nu}^{(\mu)} \sum_{w \in W_{\mathfrak{a}}} \epsilon(w) e^{w \circ\left(\nu+\rho_{\mathfrak{a}}\right)-\rho_{\mathfrak{a}}} .
\end{aligned}
$$

For the trivial $\mathfrak{g}$-module $L^{0}$ with $\mu=0$, we obtain

$$
\begin{aligned}
& \frac{\pi_{\mathfrak{a}} \circ\left(\sum_{w \in W} \epsilon(w) e^{w \circ \rho-\rho}\right)}{\pi_{\mathfrak{a}} \circ\left(\prod_{\alpha \in \Delta^{+}}\left(1-e^{-\alpha}\right)^{\operatorname{mult}(\alpha)}\right)}=\frac{\sum_{w \in W_{\mathfrak{a}}} \epsilon(w) e^{w \circ \rho_{\mathfrak{a}}-\rho_{\mathfrak{a}}}}{\prod_{\beta \in \Delta_{\mathfrak{a}}^{+}}\left(1-e^{-\beta}\right)^{\mathrm{mult}_{\mathfrak{a}}(\beta)}}, \\
& \frac{\pi_{\mathfrak{a}} \circ\left(\prod_{\alpha \in \Delta^{+}}\left(1-e^{-\alpha}\right)^{\operatorname{mult}(\alpha)}\right)}{\prod_{\beta \in \Delta_{\mathfrak{a}}^{+}}\left(1-e^{-\beta}\right)^{\operatorname{mult}_{\mathfrak{a}}(\beta)}}=\frac{\pi_{\mathfrak{a}} \circ\left(\sum_{w \in W} \epsilon(w) e^{w \circ \rho-\rho}\right)}{\sum_{w \in W_{\mathfrak{a}}} \epsilon(w) e^{w \circ \rho_{\mathfrak{a}}-\rho_{\mathfrak{a}}}} .
\end{aligned}
$$

Relation (6) takes the form

$$
\begin{aligned}
\pi_{\mathfrak{a}} \circ & \left(\sum_{w \in W} \epsilon(w) e^{w \circ(\mu+\rho)-\rho}\right) \sum_{w \in W_{\mathfrak{a}}} \epsilon(w) e^{w \circ \rho_{\mathfrak{a}}-\rho_{\mathfrak{a}}} \\
& =\pi_{\mathfrak{a}} \circ\left(\sum_{w \in W} \epsilon(w) e^{w \circ \rho-\rho}\right) \sum_{\nu \in P_{\mathfrak{a}}^{+}} b_{\nu}^{(\mu)} \sum_{w \in W_{\mathfrak{a}}} \epsilon(w) e^{w \circ\left(\nu+\rho_{\mathfrak{a}}\right)-\rho_{\mathfrak{a}}} .
\end{aligned}
$$

Introducing the anomalous branching coefficients in accordance with (4), we get

$$
\begin{aligned}
\pi_{\mathfrak{a}} \circ\left(\sum_{w \in W} \epsilon(w) e^{w \circ(\mu+\rho)-\rho}\right) & \sum_{w \in W_{\mathfrak{a}}} \epsilon(w) e^{w \circ \rho_{\mathfrak{a}}-\rho_{\mathfrak{a}}} \\
= & \pi_{\mathfrak{a}} \circ\left(\sum_{w \in W} \epsilon(w) e^{w \circ \rho-\rho}\right) \sum_{\xi \in P_{\mathfrak{a}}} k_{\xi}^{(\mu)} e^{\xi}
\end{aligned}
$$

Or

$$
\sum_{w \in W, v \in W_{\mathfrak{a}}} \epsilon(w) \epsilon(v) e^{\pi_{\mathfrak{a}} \circ(w \circ(\mu+\rho)-\rho)+v \circ \rho_{\mathfrak{a}}}-\rho_{\mathfrak{a}}=\sum_{\xi \in P_{\mathfrak{a}}} \sum_{w \in W} \epsilon(w) e^{\pi_{\mathfrak{a}} \circ(w \circ \rho-\rho)+\xi} k_{\xi}^{(\mu)} .
$$

Thus, we have proved the following statement.

Proposition 1. Let $L^{\mu}$ be the integrable module of the algebra $\mathfrak{g}$ with the highest weight $\mu$. Suppose $\mathfrak{a} \subset \mathfrak{g}, \mathfrak{h}_{\mathfrak{a}} \subset \mathfrak{h}_{\mathfrak{g}}, \mathfrak{h}_{\mathfrak{a}}^{*} \subset \mathfrak{h}_{\mathfrak{g}}^{*}$, and $\pi_{\mathfrak{a}}$ is a projection $P \longrightarrow P_{\mathfrak{a}}$. Then for any point $\xi \in P_{\mathfrak{a}}$ we have

$$
\sum_{w \in W} \epsilon(w) k_{\xi-\pi_{\mathfrak{a}} \circ(w \circ \rho-\rho)}^{(\mu)}=\sum_{w \in W, v \in W_{\mathfrak{a}}} \epsilon(w) \epsilon(v) \delta_{\pi_{\mathfrak{a}} \circ(w \circ(\mu+\rho)-\rho), \xi+\rho_{\mathfrak{a}}-v \circ \rho_{\mathfrak{a}}} .
$$

This expression can be rewritten to produce a recursion relation for the anomalous multiplicities:

(11) $k_{\xi}^{(\mu)}=-\sum_{w \in W \backslash e} \epsilon(w) k_{\xi-\pi_{\mathfrak{a}} \circ(w \circ \rho-\rho)}^{(\mu)}+\sum_{w \in W, v \in W_{\mathfrak{a}}} \epsilon(w) \epsilon(v) \delta_{\pi_{\mathfrak{a}} \circ(w \circ(\mu+\rho)-\rho), \xi+\rho_{\mathfrak{a}}-v \circ \rho_{\mathfrak{a}}}$. 
This formula can be applied to find the branching coefficients $b_{\nu}^{(\mu)}$ due to the fact that, when restricted to the fundamental Weyl chamber $\left(C_{\mathfrak{a}}\right)$, the anomalous branching coefficients coincide with the branching coefficients:

$$
k_{\xi}^{(\mu)}=b_{\xi}^{(\mu)} \quad \text { for } \quad \xi \in C_{\mathfrak{a}} .
$$

Relation (11) involves the standard system of shifts, $\xi \longrightarrow \xi-\pi_{\mathfrak{a}} \circ(w \circ \rho-\rho)$, determined by the singular weights of the trivial module, and the corresponding element of the algebra $\mathcal{E}$ is $\Psi^{(0)}=e^{\frac{|\rho|^{2}}{2 k} \delta-\rho} \times \sum_{s \in \dot{W}} \epsilon(s) \Theta_{s \circ(\rho)}$. At the same time, the second term on the right-hand side involves summation in $W$ and in $W_{\mathfrak{a}}$. Below we demonstrate that this relation can be simplified by introducing a different system of shifts.

We return to (6). The conditions $\mathfrak{a} \longrightarrow \mathfrak{g}$ and $\mathfrak{h}_{\mathfrak{a}} \subset \mathfrak{h}_{\mathfrak{g}}$ guarantee the inclusion $\Delta_{\mathfrak{a}}^{+} \subset$ $\Delta^{+}$. Thus, the first factor on the right, being an element of $\mathcal{E}$, can be written as

$$
\begin{aligned}
\frac{\pi_{\mathfrak{a}} \circ\left(\prod_{\alpha \in \Delta^{+}}\left(1-e^{-\alpha}\right)^{\operatorname{mult}(\alpha)}\right)}{\prod_{\beta \in \Delta_{\mathfrak{a}}^{+}}\left(1-e^{-\beta}\right)^{\text {mult }_{\mathfrak{a}}(\beta)}} & =\prod_{\alpha \in\left(\pi_{\mathfrak{a}} \circ \Delta^{+}\right)}\left(1-e^{-\alpha}\right)^{\operatorname{mult}(\alpha)-\operatorname{mult}_{\mathfrak{a}}(\alpha)} \\
& =-\sum_{\gamma \in P_{\mathfrak{a}}} s(\gamma) e^{-\gamma}
\end{aligned}
$$

For the coefficient function $s(\gamma)$, we define $\Phi_{\mathfrak{a} \subset \mathfrak{g}} \subset P_{\mathfrak{a}}$ as its carrier:

$$
\begin{aligned}
\Phi_{\mathfrak{a} \subset \mathfrak{g}} & =\left\{\gamma \in P_{\mathfrak{a}} \mid s(\gamma) \neq 0\right\} ; \\
\prod_{\alpha \in\left(\pi_{\mathfrak{a}} \circ \Delta^{+}\right)}\left(1-e^{-\alpha}\right)^{\text {mult }(\alpha)-\text { mult }_{\mathfrak{a}}(\alpha)} & =-\sum_{\gamma \in \Phi_{\mathfrak{a} \subset \mathfrak{g}}} s(\gamma) e^{-\gamma} .
\end{aligned}
$$

If the second factor on the right in (6) is also decomposed, then we obtain the relation

$$
\begin{aligned}
\sum_{w \in W} \epsilon(w) e^{\pi_{\mathfrak{a}} \circ(w \circ(\mu+\rho)-\rho)} & =-\sum_{\gamma \in \Phi_{\mathfrak{a} \subset \mathfrak{g}}} s(\gamma) e^{-\gamma} \sum_{\lambda \in P_{\mathfrak{a}}} k_{\lambda}^{(\mu)} e^{\lambda} \\
& =-\sum_{\gamma \in \Phi_{\mathfrak{a} \subset \mathfrak{g}}} \sum_{\lambda \in P_{\mathfrak{a}}} s(\gamma) k_{\lambda}^{(\mu)} e^{\lambda-\gamma}
\end{aligned}
$$

and the new property

$$
\sum_{w \in W} \epsilon(w) \delta_{\xi, \pi_{\mathfrak{a}} \circ(w \circ(\mu+\rho)-\rho)}+\sum_{\gamma \in \Phi_{\mathfrak{a} \subset \mathfrak{g}}} s(\gamma) k_{\xi+\gamma}^{(\mu)}=0 ; \quad \xi \in P_{\mathfrak{a}} .
$$

Thus, the following statement is true.

Proposition 2. Let $L^{\mu}$ be the integrable highest weight module of the algebra $\mathfrak{g}$. Suppose that $\mathfrak{a} \subset \mathfrak{g}, \mathfrak{h}_{\mathfrak{a}} \subset \mathfrak{h}_{\mathfrak{g}}, \mathfrak{h}_{\mathfrak{a}}^{*} \subset \mathfrak{h}_{\mathfrak{g}}^{*}$, and $\pi_{\mathfrak{a}}$ is a projection $P \longrightarrow P_{\mathfrak{a}}$. Then for any vector $\xi \in P_{\mathfrak{a}}$ the sum $\sum_{\gamma \in \Phi_{\mathfrak{a} \subset \mathfrak{g}}}-s(\gamma) k_{\xi+\gamma}^{(\mu)}$ is equal to the anomalous multiplicity of the weight $\xi$ in the module $L_{\mathfrak{g} \downarrow \mathfrak{a}}^{\mu}$.

Formula (14) also produces recursion relations for the anomalous multiplicities. Returning to relation (13), we see that $\Phi_{\mathfrak{a} \subset \mathfrak{g}}$ contains vectors with nonnegative grade and is a subset in the carrier of the singular weights element $\Psi^{(\mu)}=e^{\frac{|\mu+\rho|^{2}}{2 k} \delta-\rho} \sum_{s \in \dot{W}} \epsilon(s) \Theta_{s \circ(\mu+\rho)}$. In each grade the set $\Phi_{\mathfrak{a} \subset \mathfrak{g}}$ has finitely many vectors [5]. In particular,

$$
\#\left(\Phi_{\mathfrak{a} \subset \mathfrak{g}}\right)_{n=0}=\# \stackrel{\circ}{W} .
$$


In $\left(\Phi_{\mathfrak{a} \subset \mathfrak{g}}\right)_{n=0}$, let $\gamma_{0}$ be the lowest vector with respect to the natural ordering in $\Delta_{\mathfrak{a}}$. Decomposing the defining relation (13),

$$
\prod_{\alpha \in\left(\pi_{\mathfrak{a}} \circ \Delta^{+}\right)}\left(1-e^{-\alpha}\right)^{\text {mult }(\alpha)-\operatorname{mult}_{\mathfrak{a}}(\alpha)}=-s\left(\gamma_{0}\right) e^{-\gamma_{0}}-\sum_{\gamma \in \Phi_{\mathfrak{a} \subset \mathfrak{g} \backslash \gamma_{0}}} s(\gamma) e^{-\gamma},
$$

in (14) we obtain

$$
k_{\xi}^{(\mu)}=-\frac{1}{s\left(\gamma_{0}\right)}\left(\sum_{w \in W} \epsilon(w) \delta_{\xi, \pi_{\mathfrak{a}} \circ(w \circ(\mu+\rho)-\rho)+\gamma_{0}}+\sum_{\gamma \in \Gamma_{\mathfrak{a} \subset \mathfrak{g}}} s\left(\gamma+\gamma_{0}\right) k_{\xi+\gamma}^{(\mu)}\right),
$$

where the set

$$
\Gamma_{\mathfrak{a} \subset \mathfrak{g}}=\left\{\xi-\gamma_{0} \mid \xi \in \Phi_{\mathfrak{a} \subset \mathfrak{g}}\right\} \backslash\{0\}
$$

was introduced, called the fan of the injection $\mathfrak{a} \subset \mathfrak{g}$. Formula (16) can be viewed as a recursion relation for anomalous branching coefficients $k_{\xi}^{(\mu)}$. Contrary to (11), here the summation is only over $W$.

When $r=r_{\mathfrak{a}}$, the positive roots $\Delta_{\mathfrak{a}}^{+}$can always be chosen so that $\gamma_{0}=0$, and relation

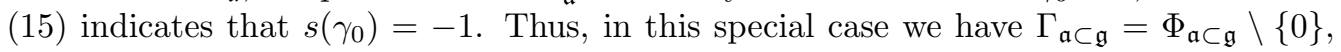
and the recursion relation takes the form

$$
k_{\xi}^{(\mu)}=\sum_{\gamma \in \Gamma_{\mathfrak{a} \subset \mathfrak{g}}} s(\gamma) k_{\xi+\gamma}^{(\mu)}+\sum_{w \in W} \epsilon(w) \delta_{\xi, \pi_{\mathfrak{a}} \circ(w \circ(\mu+\rho)-\rho)} .
$$

\subsection{Comments.}

1) The sets $\Phi_{\mathfrak{a} \subset \mathfrak{g}}$ and $\Gamma_{\mathfrak{a} \subset \mathfrak{g}}$ do not depend on the representation $L^{\mu}(\mathfrak{g})$ and describe the injection of the subalgebra $\mathfrak{a}$ into $\mathfrak{g}$.

2) Let the set of singular weights of the module $L_{\mathfrak{g} \downarrow \mathfrak{a}}^{\mu}$ be fixed. Then the sets $\Psi^{(0)}$ and $\Psi_{(\mathfrak{a})}^{(0)}$ determine the anomalous branching coefficients for the reduced module $L_{\mathfrak{g} \downarrow \mathfrak{a}}^{\mu}$ via relation (11). The same information can be obtained by using the fan $\Gamma_{\mathfrak{a} \subset \mathfrak{g}}$ via relations (16) or (18).

3) The set of branching coefficients $\left\{b_{\nu}^{(\mu)}\right\}$ is a subset of the anomalous branching coefficients $\left\{k_{\xi}^{(\mu)}\right\}$ :

$$
\left\{b_{\nu}^{(\mu)} \mid \nu \in P_{\mathfrak{a}}^{+}\right\}=\left\{k_{\xi}^{(\mu)} \mid \xi \in \overline{C_{\mathfrak{a}}}\right\} .
$$

Thus, the recursion relations (11), (16), and (18) also lead to the branching coefficients.

Now, we apply the results obtained to the case where $\mathfrak{a}$ is a Cartan subalgebra of $\mathfrak{g}$, $\mathfrak{a}=\mathfrak{h}_{\mathfrak{g}}$. Then the Weyl group $W_{\mathfrak{a}}$ and the projection $\pi_{\mathfrak{a}}$ are trivial and in formulas (11) and (18) the anomalous coefficient $k_{\xi}^{(\mu)}$ is the multiplicity of the only singular weight that contributes to the element $\Psi_{\mathfrak{h}_{\mathfrak{g}}}^{(\xi)}$, the highest weight of the $\mathfrak{h}_{\mathfrak{g}}$-submodule. This means that, in this case, the number $k_{\xi}^{(\mu)}$ is always nonnegative and coincides with the multiplicity $m_{\xi}^{(\mu)}$ of the weight $\xi$ in the module $L^{\mu}(\mathfrak{g})$. Relations (11) and (18) imply the following statement.

Corollary 3. In the integrable highest weight module $L^{\mu}(\mathfrak{g})$ of an affine Lie algebra $\mathfrak{g}$, the multiplicity $m_{\xi}^{(\mu)}$ of the weight $\xi$ (viewed as a numerical function on $P_{\mathfrak{g}}$ ) obeys the relation

$$
m_{\xi}^{(\mu)}=-\sum_{w \in W \backslash e} \epsilon(w) m_{\xi-(w \circ \rho-\rho)}^{(\mu)}+\sum_{w \in W} \epsilon(w) \delta_{(w \circ(\mu+\rho)-\rho), \xi}
$$


This enables us to construct the module $L^{\mu}(\mathfrak{g})$ recursively, provided the elements

and

$$
\Psi^{(\mu)}=e^{\frac{|\mu+\rho|^{2}}{2 k} \delta-\rho} \sum_{s \in \dot{W}} \epsilon(s) \Theta_{s \circ(\mu+\rho)}
$$

$$
\Psi^{(0)}=e^{\frac{|\rho|^{2}}{2 k} \delta-\rho} \sum_{s \in \mathfrak{W}^{\circ}} \epsilon(s) \Theta_{s \circ \rho}
$$

are known. Usually, following Racah, a truncated analog of formula (19) (without the second term on the right-hand side) is used (see, e.g., Bourbaki 13, Chapter VIII, Section 9.3]). From our point of view, relation (19) has an important advantage, because only in this form is it true for any integrable module of the category $O$ [7] and is valid for the entire lattice $P$ (outside, as well as inside, the diagram $\mathcal{N}^{\mu}$ ).

\section{$\S 4$. EXAMPLES}

Example 1. Consider the finite-dimensional Lie algebras $A_{2} \subset g_{2}$. The root system $\Delta$ is generated by a simple long root $\alpha_{1}$ and a simple short root $\alpha_{2}$ with an angle of $\frac{5 \pi}{6}$ between them:

$$
\begin{aligned}
\Delta^{+} & =\left\{\alpha_{1}, \alpha_{2}, \alpha_{1}+\alpha_{2}, \alpha_{1}+2 \alpha_{2}, \alpha_{1}+3 \alpha_{2}, 2 \alpha_{1}+3 \alpha_{2}\right\}, \\
\Delta_{s l(3)}^{+} & =\left\{\alpha_{1}, \alpha_{1}+3 \alpha_{2}, 2 \alpha_{1}+3 \alpha_{2}\right\} .
\end{aligned}
$$

By (12), (13), and (17), we obtain

$$
\begin{aligned}
& \Phi_{A_{2} \subset g_{2}}=\left\{0, \alpha_{2}, \alpha_{1}+\alpha_{2}, \alpha_{1}+3 \alpha_{2}, 2 \alpha_{1}+3 \alpha_{2}, 2 \alpha_{1}+4 \alpha_{2}\right\} \\
& \Gamma_{A_{2} \subset g_{2}}=\left\{\alpha_{2}, \alpha_{1}+\alpha_{2}, \alpha_{1}+3 \alpha_{2}, 2 \alpha_{1}+3 \alpha_{2}, 2 \alpha_{1}+4 \alpha_{2}\right\} .
\end{aligned}
$$

The singular weights of the adjoint module $L^{2 \alpha_{1}+3 \alpha_{2}}$ are

$$
\begin{array}{r}
\left\{2 \alpha_{1}+3 \alpha_{2}, 3 \alpha_{2},-\alpha_{1}+2 \alpha_{2},-4 \alpha_{2},-\alpha_{1}-6 \alpha_{2},-8 \alpha_{1}-12 \alpha_{2},-8 \alpha_{1}-13 \alpha_{2}\right. \\
\left.-6 \alpha_{1}-13 \alpha_{2},-5 \alpha_{1}-12 \alpha_{2},-6 \alpha_{1}-6 \alpha_{2},-5 \alpha_{1}-4 \alpha_{2}, 2 \alpha_{1}+2 \alpha_{2}\right\}
\end{array}
$$

and each of them corresponds to the Weyl transformation $w(\psi)$ and the quantity $\epsilon(w)$ :

$$
\{\epsilon(w(\psi))\}=\{+1,-1,+1,+1,-1,-1,+1,-1,+1,-1,+1,-1\} .
$$

In the closure of the fundamental chamber $\overline{C_{\mathfrak{a}}}$, relation (18) determines three nonzero branching coefficients

$$
b_{2 \alpha_{1}+3 \alpha_{2}}^{(\mu)}=+1, \quad b_{\alpha_{1}+2 \alpha_{2}}^{(\mu)}=+1, \quad b_{\alpha_{1}+\alpha_{2}}^{(\mu)}=+1,
$$

which correspond to the adjoint and the two fundamental submodules of $\operatorname{sl}(3)$ in the decomposition $L_{\downarrow s l(3)}^{2 \alpha_{1}+3 \alpha_{2}}$. (Notice that we need the singular weight $2 \alpha_{1}+2 \alpha_{2}$ with $s\left(2 \alpha_{1}+2 \alpha_{2}\right)=-1$ to perform the above calculations.)

Example 2. Consider the special injection of the algebra $B_{1}$ into $A_{2}$. Let $\alpha_{1}$ and $\alpha_{2}$ be the simple roots of $A_{2}$,

$$
\Delta^{+}=\left\{\alpha_{1}, \alpha_{2}, \alpha_{1}+\alpha_{2}\right\}
$$

The only positive root of $B_{1}$ is

$$
\Delta_{B_{1}}^{+}=\left\{\beta:=\frac{1}{2} \alpha_{1}\right\}
$$

Relation (12) shows that

$$
\Phi_{B_{1} \subset A_{2}}=\left\{0, \alpha_{1},-\frac{1}{2} \alpha_{1}, \frac{1}{2} \alpha_{1}\right\}=\{0,2 \beta,-\beta, \beta\} .
$$


For these vectors, the function $s_{B_{1} \subset A_{2}}$ has the values

$$
s_{B_{1} \subset A_{2}}=\{-1,+1,+1,-1\} \text {. }
$$

For the minimal vector $\gamma_{0}$, we have

$$
\begin{aligned}
\gamma_{0}^{B_{1} \subset A_{2}} & =-\beta, \\
s_{B_{1} \subset A_{2}}(-\beta) & =+1 .
\end{aligned}
$$

The fan is formed by eliminating $\gamma_{0}$ from $\Phi_{B_{1} \subset A_{2}}$ and shifting the remaining vectors by $-\gamma_{0}$ :

$$
\begin{aligned}
\Gamma_{B_{1} \subset A_{2}} & =\{\beta, 2 \beta, 3 \beta\}, \\
s_{B_{1} \subset A_{2}}\left(\gamma+\gamma_{0}\right) & =\{-,-,+\}, \quad \gamma \in \Gamma_{B_{1} \subset A_{2}} .
\end{aligned}
$$

Consider the adjoint module $L^{\alpha_{1}+\alpha_{2}}$. Its singular weights are

$$
\left\{\alpha_{1}+\alpha_{2},-\alpha_{1}+\alpha_{2},-3 \alpha_{1}-\alpha_{2},-3 \alpha_{3},-\alpha_{1}-3 \alpha_{2}, \alpha_{1}-\alpha_{2}\right\},
$$

with the values

$$
\{\epsilon(\xi)\}=\{+1,-1,+1,-1,+1,-1\} .
$$

We rewrite their projections to $P_{B_{1}}$ in terms of $\beta$ :

$$
\begin{aligned}
& \{-6 \beta,-4 \beta,-4 \beta, 0,0,+2 \beta\}, \\
\{\epsilon(\xi)\}= & \{+1,-1,+1,-1,+1,-1\} .
\end{aligned}
$$

In the closure of the fundamental chamber $\left(\overline{C_{\mathfrak{a}}}\right)$, relation (16) determines two nonzero branching coefficients

$$
b_{2 \beta}^{(\mu)}=+1, \quad b_{\beta}^{(\mu)}=+1,
$$

which correspond to the adjoint submodule and the 5-dimensional spin 2 submodule of $B_{1}$ in the reduced module $L_{\downarrow B_{1}}^{\alpha_{1}+\alpha_{2}}$. (Notice that the singular vector " 0 " with $s(0)=-1$ has multiplicity 2.)

Example 3. For the affine algebra $A_{2}^{(1)}$, consider the twisted subalgebra $A_{2}^{(2)}$. For the level $k$, the sublattice $P_{k}$ produces the normalized basis vectors $\left\{e_{1}, e_{2}, e_{3}\right\}$ with $\left|e_{j}\right|_{j=1,2,3}=1$, and $\delta$ with $|\delta|=0$. For $A_{2}^{(1)}$ we fix the simple roots

$$
\alpha_{1}=e_{1}-e_{2}, \quad \alpha_{2}=e_{2}-e_{3}, \quad \alpha_{0}=\delta-e_{1}+e_{3} .
$$

The following roots are positive:

$$
\Delta^{+}=\left\{\begin{array}{lll}
\alpha_{j}+l \delta, & j=1,2,3, & l \in \mathbf{Z}_{\geq 0}, \\
-\alpha_{j}+p \delta, & j=1,2,3, & p \in \mathbf{Z}_{>0}, \\
p \delta, & \operatorname{mult}(p \delta)=2, & p \in \mathbf{Z}_{>0}
\end{array}\right\}
$$

the classical positive roots being

$$
\stackrel{\circ}{\Delta}^{+}=\left\{\alpha_{1}=e_{1}-e_{2}, \alpha_{2}=e_{2}-e_{3}, \alpha_{3}=e_{1}-e_{3}\right\} .
$$

The fundamental weights and the Weyl vector look like this:

$$
\begin{gathered}
\omega_{1}=\frac{1}{3}\left(2 e_{1}-e_{2}-e_{3}\right)+k, \quad \omega_{2}=\frac{1}{3}\left(e_{2}+e_{1}-2 e_{3}\right)+k, \quad \omega_{0}=k ; \\
\rho=\left(\alpha_{1}+\alpha_{2}, 3,0\right) .
\end{gathered}
$$

The Weyl group is generated by the classical reflections $s_{\alpha_{1}}, s_{\alpha_{2}}$ and, in accordance with the formula $M=\sum_{i=1}^{r} \mathbf{Z} \alpha_{i}^{\vee}$ for untwisted algebras, the translations $t_{\alpha_{1}}, t_{\alpha_{2}}$.

Consider the module $L^{\omega_{0}}$. Note that, to obtain the branching rules, we only need the projected singular element $\pi_{\mathfrak{a}} \circ \Psi^{\left(\omega_{0}\right)}$ of this module and the set $\Gamma_{A_{2}^{(2)} \subset A_{2}^{(1)}}$, and we do 
not need any other properties of the module itself. We shall describe the element $\Psi^{\left(\omega_{0}\right)}$ by the set $\widetilde{\Psi^{\left(\omega_{0}\right)}}$ of singular weights of the module $L^{\omega_{0}}$. Here it has the coordinates

$$
\left\{\left(\lambda_{1}, \lambda_{2}, \lambda_{3}, n, \epsilon(w)\right) \mid \lambda_{i} \in \mathbf{Z}, n \in \mathbf{Z}_{\leq 0}, \epsilon(w)= \pm 1\right\}
$$

(the level is always $k=1$ and is not indicated). Then for $n>-10$ the set $\widehat{\Psi^{\left(\omega_{0}\right)}}$ contains the following 54 vectors:

$$
\begin{aligned}
\widehat{\Psi^{\left(\omega_{0}\right)}=} & \{(0,0,0,0,-1),(-1,1,0,0,1),(0,-1,1,0,1),(-1,-1,2,0,-1), \\
& (-2,1,1,0,-1),(-2,0,2,0,1),(2,-3,1,-2,-1),(-4,0,4,-2,-1), \\
& (-4,3,1,-2,1),(-1,-3,4,-2,1),(-1,3,-2,-2,-1),(2,0,-2,-2,1), \\
& (-5,1,4,-3,1),(-4,-1,5,-3,1),(0,-4,4,-3,-1),(-2,-3,5,-3,-1), \\
& (-4,4,0,-3,-1),(-5,3,2,-3,-1),(3,-3,0,-3,1),(2,-4,2,-3,1), \\
& (0,3,-3,-3,1),(-2,4,-2,-3,1),(3,-1,-2,-3,-1),(2,1,-3,-3,-1), \\
& (-5,-1,6,-5,-1),(-6,1,5,-5,-1),(0,-5,5,-5,1),(-2,-4,6,-5,1), \\
& (-5,5,0,-5,1),(-6,4,2,-5,1),(4,-4,0,-5,-1),(3,-5,2,-5,-1), \\
& (0,4,-4,-5,-1),(-2,5,-3,-5,-1),(3,1,-4,-5,1),(4,-1,-3,-5,1), \\
& (-6,0,6,-6,1),(-1,-5,6,-6,-1),(-6,5,1,-6,-1),(4,-5,1,-6,1), \\
& (-1,5,-4,-6,1),(4,0,-4,-6,-1),(-4,-4,8,-9,-1),(-5,-3,8,-9,1), \\
& (-8,4,4,-9,-1),(-8,3,5,-9,1),(3,-7,4,-9,1),(2,-7,5,-9,-1), \\
& (-4,7,-3,-9,1),(-5,7,-2,-9,-1),(6,-3,-3,-9,-1),(6,-4,-2,-9,1), \\
& (3,3,-6,-9,-1),(2,4,-6,-9,1), \ldots\} .
\end{aligned}
$$

The $\pi_{\mathfrak{a}}$ projection leads to the set of vectors shown in Figure 1.

In the figure the grade values increase to the right. The vertical line is the basic root vector $\beta$ of the algebra $A_{2}^{(2)}$.

For the subalgebra $A_{2}^{(2)}$, the basic roots are

$$
\beta=(1,0,0), \quad \beta_{0}=\delta-2 \beta=(-2,0,1),
$$

with

$$
\theta=2 \beta
$$

and the normalization

$$
|\beta|^{2}=1, \quad\left|\beta_{0}\right|^{2}=4, \quad\left(\beta_{0}, \beta\right)=-2 .
$$

The fundamental weights and the Weyl vector look like this:

$$
\begin{gathered}
\omega_{1}=1 / 2 \beta+k=(1 / 2,1,0), \quad \omega_{0}=2 k=(0,2,0) ; \\
\rho=1 / 2 \beta+3 k=(1 / 2,3,0) .
\end{gathered}
$$

The positive roots

$$
\Delta_{A_{2}^{(2)}}^{+}=\left\{\begin{array}{ll}
\beta+n \delta, \pm 2 \beta+(2 n+1) \delta ; & n \in \mathbf{Z}_{\geq 0} \\
-\beta+m \delta ; \quad m \delta ; & m \in \mathbf{Z}_{>0}
\end{array}\right\}
$$

have multiplicity one. The Weyl group $W_{A_{2}^{(2)}}$ is generated by the classical reflection $s_{\beta}$ and the translations $t \in T_{A_{2}^{(2)}} \subset W_{A_{2}^{(2)}}$ along the coroot $\alpha_{0}^{\vee}=1 / 2 \delta-\beta=(-1,0,1 / 2)$ : $T_{A_{2}^{(2)}}=\left\{t_{l \alpha_{0}^{\vee}}, l \in \mathbf{Z}\right\}$. 


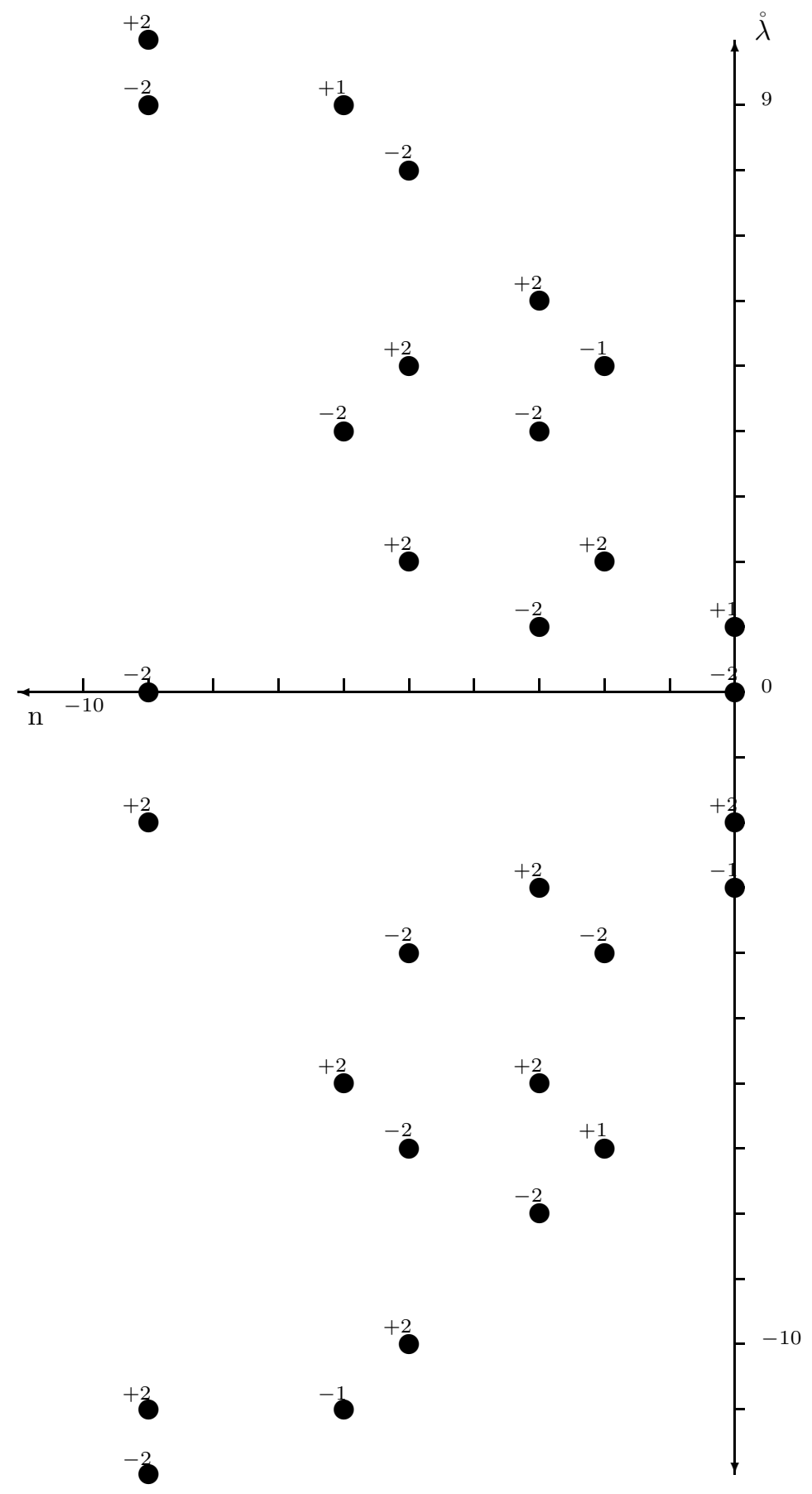

FiguRE 1

The injection $A_{2}^{(2)} \longrightarrow A_{2}^{(1)}$ is governed by its classical part, which is the special injection $B_{1} \longrightarrow A_{2}$. The latter means that, when we construct the subset $\Delta_{A_{2}^{(2)}}$ in the root space of $A_{2}^{(1)}$, the roots in $\Delta_{A_{2}^{(2)}}$ are rescaled:

$$
\beta=\alpha_{1} / 2, \quad K_{A_{2}^{(2)}}=2 K_{A_{2}^{(1)}} .
$$


(So, in the modules $L^{\mu}$ of level $k$ the $A_{2}^{(2)}$-submodules have level $2 k$.)

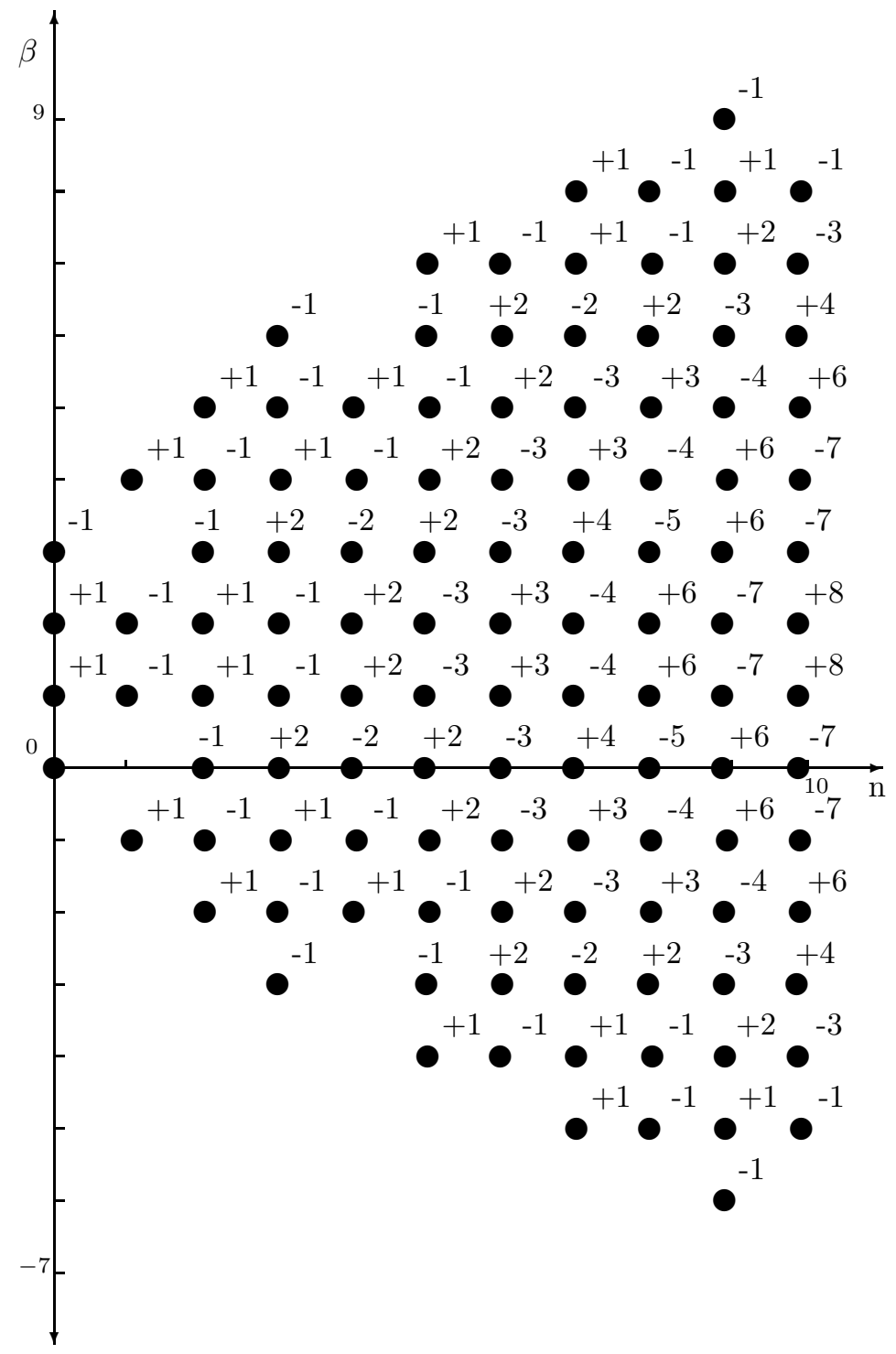

Figure 2

In accordance with (13), the set $\Phi_{A_{2}^{(2)} \subset A_{2}^{(1)}}$ is determined by the opposite vectors in the nonzero components of the element $\prod_{\alpha \in\left(\pi_{A_{2}^{(2)}}{ }^{\circ} \Delta^{+}\right)}\left(1-e^{-\alpha}\right)^{\text {mult }(\alpha)-\text { mult }_{\mathfrak{a}}(\alpha)}$. Taking the rescaling (38) into account, we obtain the element

$$
\left(1-e^{-\beta}\right)\left(1-e^{2 \beta}\right) \times \prod_{\varkappa= \pm 1} \prod_{n=1}^{\infty}\left(1-e^{\varkappa 2 \beta+2 n \delta}\right)\left(1-e^{\varkappa \beta+n \delta}\right) \prod_{m=1}^{\infty}\left(1-e^{+m \delta}\right)
$$

generated by the set $\Phi_{A_{2}^{(2)} \subset A_{2}^{(1)}}$. Here the lowest vector $\gamma_{0}$ is $-\beta$, and the fan is

$$
\Gamma_{A_{2}^{(2)} \subset A_{2}^{(1)}}=\left\{\xi+\beta \mid \xi \in \Phi_{A_{2}^{(2)} \subset A_{2}^{(1)}}\right\} \backslash\{0\} .
$$


The structure of the fan can be illustrated by Figure 2, which presents the vectors $\gamma \in \Gamma_{A_{2}^{(2)} \subset A_{2}^{(1)}}$ and their multiplicities $s(\gamma)$ with grade $n \leq 10$.

Now we are able to construct the branching rules and explicitly reduce the module $L^{\omega_{0}}$ with respect to the subalgebra $A_{2}^{(2)}$. Note that, in terms of $A_{2}^{(2)}$, the diagram $\mathcal{N}^{\omega_{0}}$ is located in the subspace of level $k=2$. Applying formula (16) in the sublattice with $n=0$, we find the first nontrivial value for the weight with the highest (for $n=0$ ) anomalous multiplicity (that is, for the vector $(1,2,0)$ ), where obviously, the branching coefficient is $k_{(1,2,0)}^{(\mu)}=1$. The recursion procedure generates the set $\widehat{\Psi_{A_{2}^{(2)}}}$ (see Figure 3 ).

We have presented the branching in terms of the singular weights $\overline{\Psi_{A_{2}^{(2)}}^{(\xi)}}$ of the submodules $L_{A_{2}^{(2)}}^{\xi}$ in the decomposition

$$
L_{A_{2}^{(1)} \downarrow A_{2}^{(2)}}^{\mu}=\bigoplus_{\xi \in P_{A_{2}^{(2)}}^{+}} b_{\xi}^{(\mu)} L_{A_{2}^{(2)}}^{\xi} .
$$

Now it is quite easy to extract the branching coefficients $b_{\xi}^{(\mu)}$. The intersection

$$
\widehat{\Psi_{A_{2}^{(2)}}} \cap \bar{C}_{A_{2}^{(2)}}
$$

gives the set of highest weights and their multiplicities $b_{\xi}^{(\mu)}$, and the branching is

$$
\begin{aligned}
L_{A_{2}^{(1)} \downarrow A_{2}^{(2)}}^{\omega_{0}}=L_{A_{2}^{(2)}}^{\omega_{0}}(0) & \oplus L_{A_{2}^{(2)}}^{2 \omega_{1}}(-1) \oplus 2 L_{A_{2}^{(2)}}^{2 \omega_{1}}(-3) \oplus L_{A_{2}^{(2)}}^{\omega_{0}}(-4) \\
& \oplus 2 L_{A_{2}^{(2)}}^{2 \omega_{1}}(-5) \oplus 2 L_{A_{2}^{(2)}}^{\omega_{0}}(-6) \oplus 4 L_{A_{2}^{(2)}}^{2 \omega_{1}}(-7) \\
& \oplus 3 L_{A_{2}^{(2)}}^{\omega_{0}}(-8) \oplus 5 L_{A_{2}^{(2)}}^{2 \omega_{1}}(-9) \oplus 4 L_{A_{2}^{(2)}}^{\omega_{0}}(-10) \oplus \cdots
\end{aligned}
$$

(Note that, since we have shifted the set $\Phi_{A_{2}^{(2)} \subset A_{2}^{(1)}}$, the Weyl chamber $\bar{C}_{A_{2}^{(2)}}$ is also shifted accordingly.) The result can be presented in terms of two branching functions

$$
\begin{aligned}
& b_{I}^{(\mu)}(q)=1+q^{4}+2 q^{6}+3 q^{8}+4 q^{10}+\cdots \\
& b_{I I}^{(\mu)}(q)=q+2 q^{3}+2 q^{5}+4 q^{7}+5 q^{9}+\cdots .
\end{aligned}
$$

\section{$\S 5$. Conclusions}

We have demonstrated that the decompositions of integrable highest weight modules of simple Lie algebras (classical or affine) with respect to their reductive subalgebras is subject to an (infinite) set of algebraic relations. These relations originate from the properties of singular vectors of the module $L_{\mathfrak{g}}$, viewed as the highest weights of the Verma modules $M_{\mathfrak{a}}$. Thus, it becomes clear that each property involves a finite set of weights, where the minimal element is fixed by the higher ones. This gives rise to recursion relations for branching coefficients.

The properties stated above are encoded in the subset $\Gamma_{\mathfrak{g} \supset \mathfrak{a}}$ of the weight lattice $P_{\mathfrak{a}}$, called the fan of the injection. The fan depends only on the map $\mathfrak{a} \longrightarrow \mathfrak{g}$. It describes the injection (no matter regular or special) precisely as the root system describes the injection $\mathfrak{h}(\mathfrak{g}) \longrightarrow \mathfrak{g}$ of the Cartan subalgebra. Thus, in the simple case where $\mathfrak{a}=\mathfrak{h}(\mathfrak{g})$, the recursion procedure generates the weight diagram of the module $L_{\mathfrak{g}}$.

When applied to a reduction of highest weight modules, the recursion described by a fan provides a highly efficient tool for obtaining the explicit values of the branching coefficients. 

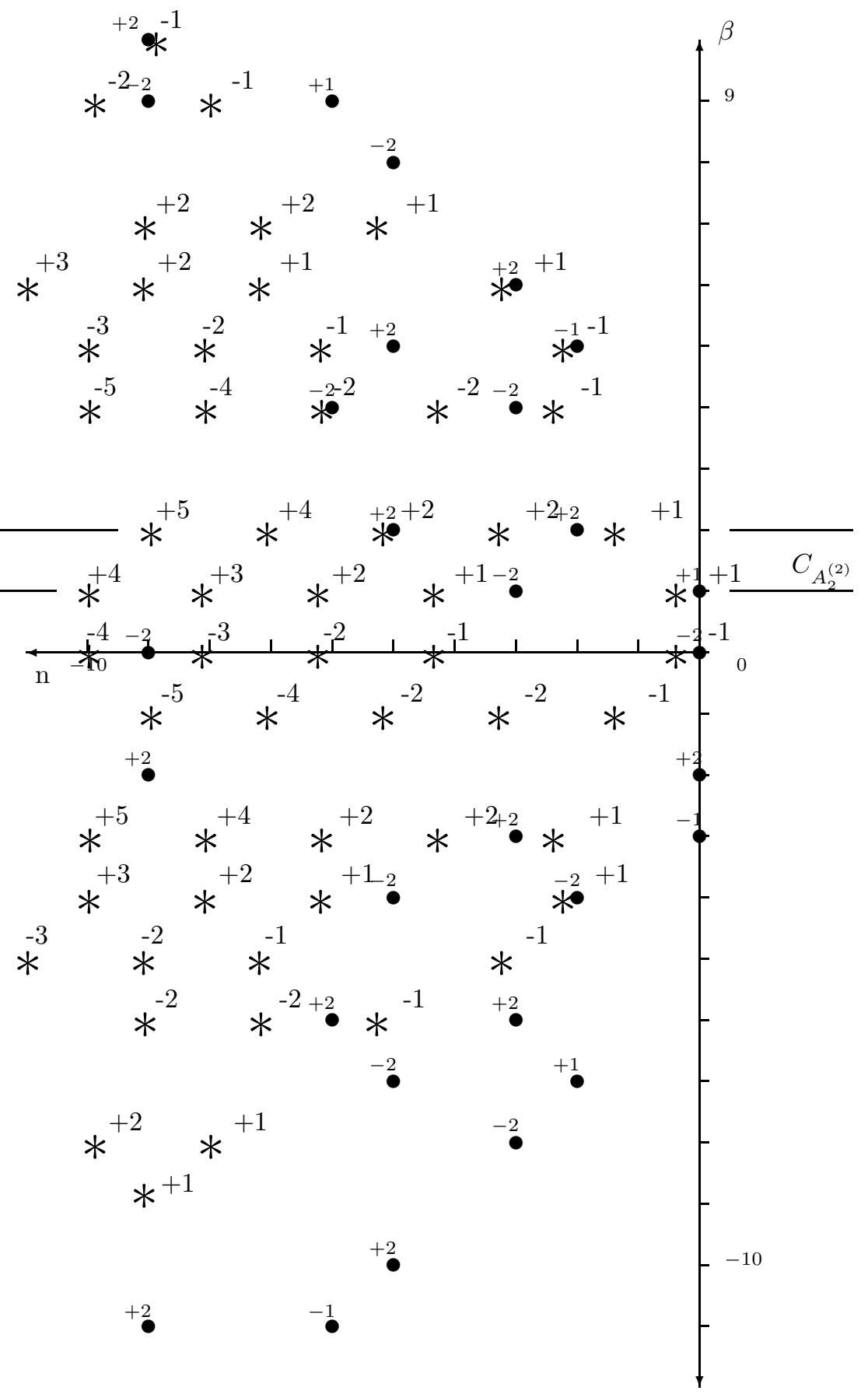

FiguRE 3

\section{REFERENCES}

[1] E. Date, M. Jimbo, A. Kuniba, T. Miwa, and M. Okado, One-dimensional configuration sums in vertex models and affine Lie algebra characters, Preprint RIMS-631, 1988; Lett. Math. Phys. 17 (1989), no. 1, 69-77. MR0990586 (90c:82052)

[2] L. D. Faddeev, How the algebraic Bethe ansatz works for integrable models, Quantum Symmetries/Symétries Quantiques (Les Houches, 1995), North-Holland, Amsterdam, 1998, pp. 149-219. MR1616371 (2000b:82010) 
[3] V. A. Kazakov and K. Zarembo, Classical/quantum integrability in non-compact sector of $A d S / C F T$, J. High Energy Phys. 2004, no. 10, 060, 23 pp. (electronic). MR2115170 (2005h:81294)

[4] N. Beisert, The dilatation operator of $N=4$ super Yang-Mills theory and integrability, Phys. Rep. 405 (2004), 1-202. MR2109451 (2006b:81302)

[5] V. Kac, Infinite-dimensional Lie algebras, Cambridge Univ. Press, Cambridge, 1990. MR1104219 (92k:17038)

[6] M. Wakimoto, Infinite-dimensional Lie algebras, Transl. Math. Monogr., vol. 195, Amer. Math. Soc., Providence, RI, 2001. MR.1793723 (2001k:17038)

[7] I. N. Bernstein, I. M. Gel'fand, and S. I. Gel'fand, Differential operators on the basic affine space and a study of $\gamma$-modules, Lie groups and their representations (Proc. Summer School, Bolyai János Math. Soc., Budapest, 1971), Halsted Press, New York, 1975, pp. 21-64. MR0578996 (58:28285)

[8] B. Fauser, P. D. Jarvis, R. C. King, and B. G. Wybourn, New branching rules induced by plethysm, J. Phys. A 39 (2006), no. 11, 2611-2655. arXiv:math-RT/0308043, 2003. MR2213359 (2007j:05211)

[9] S. Hwang and H. Rhedin, General branching functions of affine Lie algebras, Modern Phys. Lett. A 10(1995), no. 10, 823-830, arXiv:hep-th/ 9408087, 1994. MR.1329673 (96c:17038)

[10] B. Feigin, E. Feigin, M. Jimbo, T. Miwa, and E. Mukhin, Principal $\mathfrak{s l}_{3}$ subspaces and quantum Toda Hamiltonians, arXiv:0707.1635v2, 2007.

[11] V. D. Lyakhovsky and S. Yu. Melnikov, Recursion relations and branching rules for simple Lie algebras, J. Phys. A 29 (1996), 1075-1087. MR1383070 (97c:17007)

[12] V. D. Lyakhovsky, Recurrent properties of affine Lie algebra representations, Supersymmetry and Quantum Symmetry, Dubna (to appear).

[13] N. Bourbaki, Éléments de mathématique. Fasc. 38. Groupes et algèbres de Lie, Actualités Sci. Indust., No. 1364, Hermann, Paris, 1975. MR0453824 (56:12077)

Department of Theoretical Physics, St. Petersburg State University, St. Petersburg 198904, RUSSIA

E-mail address: milyin-5@mail.ru

St. Petersburg Branch, Steklov Mathematical Institute, Russian Academy of Sciences, Fontanka 27, St. Petersburg 191023, Russia

E-mail address: kulish@pdmi.ras.ru

Department of Theoretical Physics, St. Petersburg State University, St. Petersburg 198904, RUSSIA

E-mail address: lyakh1507@nm.ru

Received 14/SEP/2008

Translated by THE AUTHORS 